\title{
Sample Cleanup Using Solid-Phase Dispersive Extraction for Determination of Vancomycin in Serum
}

\author{
Yasuhiro Sakamoto, Yuki Jinno, Ikumi Shinodzuka, Yusuke IwaSaki, Rie Ito, and Koichi SaIto \\ Department of Analytical Chemistry, Faculty of Pharmaceutical Sciences, Hoshi University, 2-4-41 Ebara, \\ Shinagawa, Tokyo 142-8501, Japan
}

\begin{abstract}
A cleanup method employing quick and simple solid-phase dispersive extraction (SPDE) was investigated for its potential use in the determination of vancomycin (VCM) in serum by liquid chromatography/mass-spectrometry (LC/MS). SPDE was observed to be more rapid than conventional cartridge-type solid-phase extraction (SPE). In addition, in the analysis of viscous samples such as serum containing many proteins, SPDE could satisfactorily remove proteins even if deproteinization was not performed beforehand. The limit of detection $(S / N=3)$ and the limit of quantification $(S / N>$ 10) of VCM by LC/MS were 0.05 and $0.2 \mathrm{ng} / \mathrm{mL}$, respectively. The average recoveries of VCM from pooled serum spiked at 2,10 , and $100 \mathrm{ng} / \mathrm{mL}$ were $90.0,90.8$, and $98.6 \%$, respectively. The repeatabilities were $7.5,6.8$, and $2.8 \%$, and the intermediate precision values were $8.5,6.8$, and $7.0 \%$, respectively. This suggests that the developed analytical method combing SPDE is useful for the determination of VCM in serum.
\end{abstract}

Keywords Solid-phase dispersive extraction, vancomycin, serum, LC/MS

(Received September 17, 2013; Accepted November 25, 2013; Published February 10, 2014)

\section{Introduction}

Vancomycin (VCM, Fig. 1) is a glycopeptide antibiotic produced by Amycolatopsis orientalis. It is used for the treatment of infectious diseases caused by methicillin-resistant Staphylococcus aureus. However, VCM has a narrow therapeutic range $\left(10-20 \mu \mathrm{g} / \mathrm{mL}\right.$ in whole blood) at trough levels. ${ }^{1,2}$ Serious side effects such as nephrotoxicity and acoustic disturbance could occur when the VCM concentration in the blood exceeds $20 \mu \mathrm{g} / \mathrm{mL}$ at the trough level. ${ }^{3,4}$ The rapid elevation in the circulating levels of VCM has the potential for causing many side effects (e.g., red neck syndrome and hypotension). ${ }^{5}$ In fact, a serious medical accident recently occurred in Japan, which involved the necrosis of an infant's toe, because the infant was mistakenly injected with an excessive dose of VCM. ${ }^{6}$ Therefore, therapeutic drug monitoring (TDM) was recommended for the efficacious and fair use of VCM.

TDM is a method for performing risk management (fair use and side effect revelation), and it is applied to many medicines (e.g., antibacterial and immunosuppressive drugs). ${ }^{7,8} \mathrm{~A}$ convenient fluorescence polarization immunoassay (FPIA) was widely used in TDM for measuring blood concentration. ${ }^{9,10}$ However, FPIA has low accuracy and precision because FPIA is susceptible to fluctuations in the temperature and viscosity of the solvent. And FPIA might be unable to correctly measure blood concentrations. Therefore, the accurate determination of blood concentration was considered important for TDM. Some physicochemical methods such as liquid chromatography/ ultraviolet spectrometry (LC/UV), sensitive fluorometry (FL),

† To whom correspondence should be addressed.

E-mail: k-saito@hoshi.ac.jp and mass spectrometry (MS) were also used to correctly determine blood concentration. ${ }^{11-15}$ However, a sample cleanup procedure (deproteinization extraction and solid-phase extraction (SPE)) is indispensable for eliminating the impurities from the sample matrix when performing such instrumental analysis. ${ }^{16,17}$ The conventional cartridge-type SPE method has some drawbacks, such as clogging of the sample solution in cases of high-viscosity biological samples, which results in low

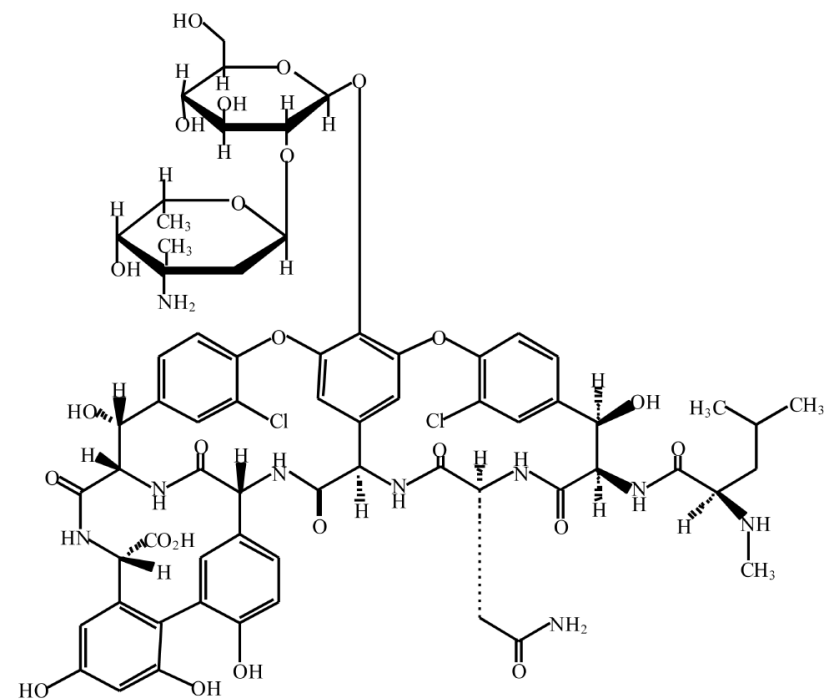

Vancomycin

Fig. 1 Chemical structure of VCM. 
recoveries. In addition, it was difficult to maintain a constant flow rate for all SPE cartridges when a vacuum-type manifold was used for conventional cartridge-type SPE operation. Therefore, we constructed simple and rapid solid-phase dispersive extraction (SPDE) as a cleanup method, which overcomes the described drawbacks for the determination of benzodiazepines in serum and urine. ${ }^{18}$ SPDE is a method of rapidly extracting target compounds by dispersing microparticles into a liquid sample. A commercially available reversed-phase polymer-gel was adopted as the solid-phase gel for SPDE. In conventional liquid-liquid extraction (or solidliquid extraction), compound extractability from the phase containing a compound to another phase usually depends on a distribution coefficient. For rapid equilibrium, vigorous mixing (shaking or stirring) is necessary. On the other hand, when solid-phase microparticles are dispersed in a liquid solution, it seems that equilibrium between the two phases of the suspending solution was reached immediately after dispersion. In other words, adsorption and desorption to the solid-phase gel occurred almost instantaneously for SPDE. The variation in recoveries among samples could be decreased owing to processing of many samples by centrifugation. Further, SPDE seemed to be barely affected by exposure to infectious and/or chemically hazardous samples because SPDE operation is performed in a closed system. The determination of benzodiazepines in serum and urine ${ }^{18}$ produced excellent results for cleanup using SPDE after deproteinization extraction in the determination of benzodiazepines in serum.

In this study, the deproteinization ability of SPDE was newly evaluated because we found that SPDE has sufficient deproteinization ability. Residual amounts of proteins in serum after SPDE cleanup were measured by the BCA method, ${ }^{22,23}$ and the deproteinization ability of SPDE was compared with that using a deproteinization reagent such as trichloroacetic acid aqueous solution (TCA), methanol, and acetonitrile. In addition, SPDE was compared with conventional cartridge-type SPE ${ }^{19-21}$ regarding the operation time and recovery rates to evaluate its usefulness, and VCM in serum was measured by LC/MS.

\section{Experimental}

\section{Materials and reagents}

CONSERA $^{\circledR}$ "Nissui" pooled serum sample was purchased from Nissui Pharmaceutical Co., Ltd. (Tokyo, Japan).

VCM standard was purchased from Wako Pure Chemical Industries, Ltd. (Osaka, Japan). The VCM standard stock solution $(1.0 \mathrm{mg} / \mathrm{mL})$ was prepared with water. Working solutions were prepared by diluting the stock solution with water to achieve the appropriate concentrations. The VCM standard solutions were stored at $4^{\circ} \mathrm{C}$ until use.

LC-grade methanol and acetonitrile were special-grade acetic acid and TCA were purchased from Wako Pure Chemical Industries, Ltd.. Water was purified with a Milli-Q Gradient A10 system equipped with an EDS-PAK ${ }^{\mathrm{TM}}$ polisher (Merck Millipore Ltd., Bedford, MA). A silicon antifoaming agent was purchased from Merck Millipore Ltd.

An Oasis ${ }^{\circledR}$ HLB cartridge (30 $\mu \mathrm{m}$ o.d.; Waters Co., Milford, MA) was used for SPE, and after taking the solid-phase gel from the corresponding Oasis ${ }^{\circledR}$ HLB SPE cartridge, the gel was used for SPDE. We purchased @Roka ${ }^{\mathrm{TM}}$ and Captube ${ }^{\mathrm{TM}}$ centrifugation filter units from Frontier Science Co., Ltd. (Hokkaido, Japan).

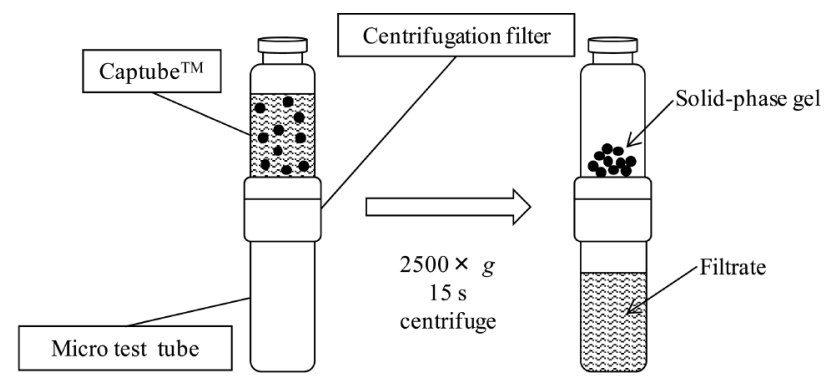

Fig. 2 Scheme of SPDE using a centrifugation filter.

\section{Apparatus}

LC/MS analysis was performed with Agilent 1100 Series LC-MSD systems (Agilent Technologies, Inc., CA). The LC separation of VCM was performed with a ZIC ${ }^{\circledR}$-HILIC column ( $2.1 \mathrm{~mm}$ i.d. $\times 100 \mathrm{~mm}, 5 \mu \mathrm{m}$; Merck SeQuant AB, Sweden). A mixture of acetonitrile/water as the mobile phase was delivered at a flow rate of $0.2 \mathrm{~mL} / \mathrm{min}$ with the linear gradient described below. The gradient condition was acetonirile-water (90:10, v/v) for $0-3 \mathrm{~min}$, acetonirile-water $(90: 10, \mathrm{v} / \mathrm{v}-30: 70$, $\mathrm{v} / \mathrm{v})$ from 3 to $6 \mathrm{~min}$, acetonirile-water $(30: 70, \mathrm{v} / \mathrm{v})$ for 6 $10 \mathrm{~min}$, acetonirile-water $(30: 70, \mathrm{v} / \mathrm{v}-10: 90, \mathrm{v} / \mathrm{v})$ from 10 to $11 \mathrm{~min}$, acetonirile-water $(10: 90, \mathrm{v} / \mathrm{v})$ for $11-14 \mathrm{~min}$, and acetonirile-water (10:90, v/v - 90:10, v/v) from 14 to $15 \mathrm{mn}$.

The working parameters for LC/MS were as follows: ionization mode, positive ion electrospray ionization (ESI); monitoring ion, $\mathrm{m} / \mathrm{z} 725\left([\mathrm{M}+2 \mathrm{H}]^{2+}\right)$; nebulizer gas and drying gas, nitrogen; nebulizer gas pressure, $50 \mathrm{psi}$; drying gas flow rate, $11 \mathrm{~L} / \mathrm{min}$; drying gas temperature, $350^{\circ} \mathrm{C}$ : capillary voltage, $3000 \mathrm{~V}$; fragment voltage, $120 \mathrm{~V}$.

\section{Sample preparation}

SPDE operation was performed according to our previous method. ${ }^{18}$ Briefly, the Oasis ${ }^{\circledR}$ HLB solid-phase gel taken from the corresponding SPE cartridge was dispersed in water at a concentration of $100 \mathrm{mg} / \mathrm{mL}$, preceded by conditioning with water and methanol. A Captube ${ }^{\mathrm{TM}}$ was set on the top of $@ \mathrm{Roka}^{\mathrm{TM}}$, and a conventional 2.0-mL micro test tube was attached to the bottom of the @Roka ${ }^{\mathrm{TM}}$ filter unit (Fig. 2). The pooled serum sample $(500 \mu \mathrm{L})$ was applied to the Captube ${ }^{\mathrm{TM}}$. Then, $500 \mu \mathrm{L}$ of water and a single drop of a silicon antifoaming agent were added to the pooled serum in the Captube ${ }^{\mathrm{TM}}$. Subsequently, $100 \mu \mathrm{L}$ of the Oasis ${ }^{\circledR}$ HLB solid-phase gel suspension $(100 \mathrm{mg} / \mathrm{mL})$ was added to the pooled serum in a similar manner. The solid-phase gel suspension was immediately agitated for $10 \mathrm{~s}$ with a vortex mixer to sufficiently disperse the solid-phase gel in the pooled serum sample. The centrifugal filter unit was centrifuged $(2500 \times g, 15 \mathrm{~s})$, and the filtrate in the micro test tube attached at the bottom of the @ Roka ${ }^{\mathrm{TM}}$ filter unit was eliminated together with the micro test tube itself. To wash the solid-phase gel, $1 \mathrm{~mL}$ of water was added to the Captube ${ }^{\mathrm{TM}}$ and the solid-phase gel was dispersed once again. Subsequently, the solvent phase was eliminated as described above. In addition, the washing step was repeated two times. Next, $1 \mathrm{~mL}$ of a $60 \%$ methanol aqueous solution was added to the Captube ${ }^{\mathrm{TM}}$, the solid-phase gel was dispersed and centrifuged $(2500 \times \mathrm{g}$, $15 \mathrm{~s}$ ) once again for eluting VCM. The eluting operation was repeated two times. The eluates were combined, and the total volume was made up to $2 \mathrm{~mL}$. A $50-\mu \mathrm{L}$ aliquot of the eluates was injected into the LC/MS instrument. 


\section{Determination of proteins in the serum}

A commercial BCA assay kit (Pierce ${ }^{\mathrm{TM}}$ BCA Protein Assay Kit, Thermo Fisher Scientific K.K.) was used for the determination of proteins in the serum. The BCA method was operated according to the manufacturer's instructions.

First, the provided solution A (sodium carbonate, sodium bicarbonate, bicinchoninic acid, and sodium tartrate containing a $0.1 \mathrm{M}$ sodium hydroxide aqueous solution) was mixed with the other provided solution B (a 4\% copper sulfate pentahydrate aqueous solution) in a ratio of 50:1; this mixture is called BCA reagent. The following seven samples were used in the experiment. Namely, the pooled serum was (1) prepared by the procedure described above, (2) diluted with water, (3) deproteinized by a $1 \%$ TCA, (4) deproteinized by $5 \%$ TCA, (5) deproteinized by $10 \%$ TCA, (6) deproteinized by $5 \%$ methanol, and (7) deproteinized by acetonitrile. The prepared samples (1), (6), and (7) were dried to remove residual organic solvent by nitrogen purge, and the residue was redissolved in water before measurement by the BCA method. The $\mathrm{pH}$ levels of samples (3), (4), and (5) were nearly neutralized with a sodium hydroxide aqueous solution before measurement by the BCA method. Finally, the volume of each sample solution was adjusted to $2 \mathrm{~mL}$. Each sample was added in steps of $25 \mu \mathrm{L}$ to each well of a 96-well microplate. Subsequently, $200 \mu \mathrm{L}$ of the BCA reagent was added to each well of the 96-well microplate and mixed for $30 \mathrm{~s}$. The reaction was carried out for $2 \mathrm{~h}$ at room temperature. The absorbance of each sample was measured at $562 \mathrm{~nm}$ using a microplate reader (Model 550, Bio-Rad Laboratories, Inc.).

\section{Results and Discussion}

\section{LC conditions}

The optimum dissolving solvent for a VCM standard solution was investigated to skip the nitrogen purge operation of the eluate after SPDE. The peak area of VCM dissolved in $60 \%$ methanol (the elution solvent for SPDE) decreased in comparison with that in water when the determination was carried out using an L-column 2 ODS $(2.1 \mathrm{~mm}$ i.d. $\times 150 \mathrm{~mm}, 3 \mu \mathrm{m}$, Chemicals Evaluation and Research Institute, Japan). On the other hand, when a hydrophilic interaction chromatography (HILIC) column was used, the peak area of VCM was not affected by the methanol concentration in the sample solution. Thus, ZIC $^{\circledR}$-HILIC $(2.1 \mathrm{~mm}$ i.d. $\times 100 \mathrm{~mm}, 5 \mu \mathrm{m})$ was adopted as the analysis column in this study. As a result, the nitrogen purge operation to dry the eluate was omitted, and the time to complete the whole determination was shortened to about $1 \mathrm{~h}$.

\section{Analysis method validation}

The limit of detection $(S / N=3)$ and the limit of quantification $(S / N>10)$ of VCM were 0.05 and $0.2 \mathrm{ng} / \mathrm{mL}$ by LC/MS, respectively. The calibration curve showed good linearity over the range from 0.2 to $50 \mathrm{ng} / \mathrm{mL}$ with a correlation coefficient of greater than 0.999 . Thus, the therapeutic range $(10-20 \mu \mathrm{g} / \mathrm{mL})$ of VCM in TDM could be sufficiently measured with the proposed LC/MS procedure.

\section{Analysis of deproteinizing extraction}

The amount of residual proteins after the SPDE cleanup method was measured by the BCA method ${ }^{22,23}$ because SPDE was performed without deproteinization. As a result, few residual proteins were found in the solution after SPDE cleanup. It was inferred that SPDE had sufficient deproteinization ability as compared with conventional deproteinization methods using a deproteinization reagent (10\% TCA and acetonitrile) (Fig. 3).

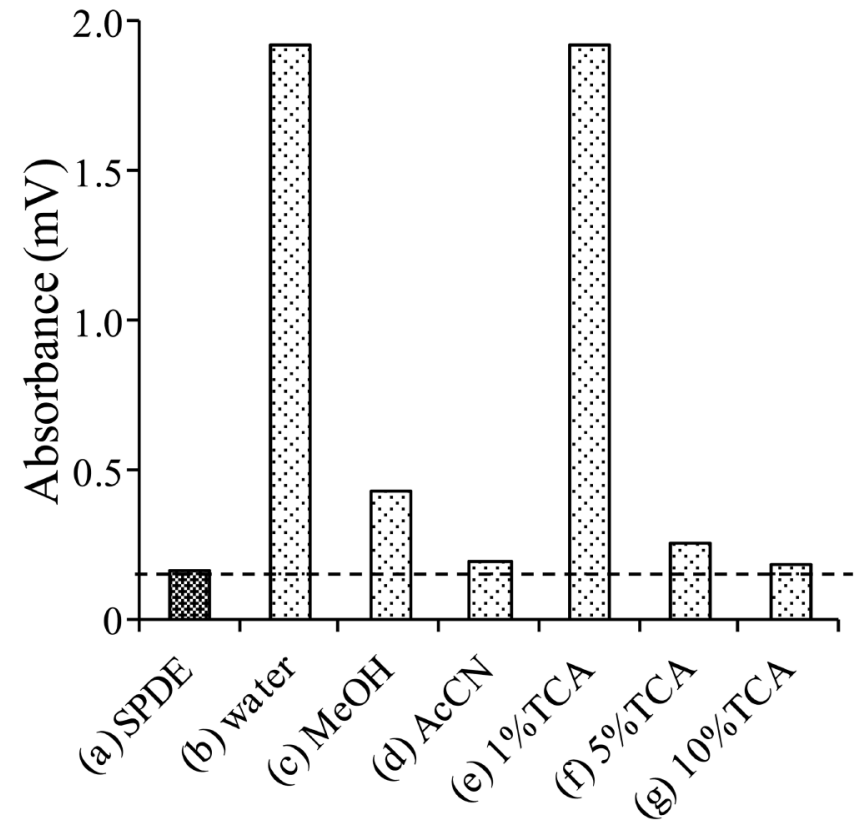

Fig. 3 Comparison of the deproteination effect on the absorbance of the BCA method among (a) SPDE, (b) dilution by water and deproteinization agent such as (c) methanol, (d) acetonitrile, (e) $1 \%$ TCA, (f) $5 \%$ TCA, and (g) $10 \%$ TCA.

Therefore, SPDE could sufficiently eliminate proteins without any deproteinization pretreatment. It was inferred that VCM in a serum sample was selectively retained by the solid-phase gel, because the affinity between the solid-phase gel and VCM was stronger than that between proteins and VCM. These results indicate that the proposed SPDE cleanup method did not necessitate any deproteinization.

\section{Optimization of SPDE conditions}

The type and amount of the solid-phase gel and the types, concentration, and amount of organic solvent for elution were investigated to optimize the SPDE operating conditions. Oasis ${ }^{\circledR}$ HLB, MCX, WCX, MAX, and WAX solid-phase gels were tested (Fig. 4) because VCM is an amphoteric compound with a carboxyl group and amino groups. Therefore, VCM was sufficiently retained by Oasis ${ }^{\circledR}$ HLB and MCX, with relatively high recoveries. In addition, Oasis ${ }^{\circledR}$ HLB was adopted as a solid-phase gel for SPDE, because the variation in recoveries with the use of Oasis ${ }^{\circledR}$ HLB was lower than that with the use of Oasis $^{\circledR}$ MCX.

Subsequently, the optimal amount of solid-phase gel was investigated within the range of $5-30 \mathrm{mg}$. As a result, recoveries of about $95 \%$ were obtained with all the amounts of solid-phase gel tested without significant differences. Therefore, $10 \mathrm{mg}$ was adopted as the optimal amount of solid-phase gel for SPDE, assuming that a real sample contains many impurities.

Methanol and acetonitrile were examined as organic solvents for the elution process of SPDE. Methanol was used as the elution solvent because the variation in recoveries with the use of methanol was lower than that with the use of acetonitrile. Next, the optimal concentration of methanol and the volume of eluting solution were examined within the range of $10-100 \%$. A $60 \%$ methanol aqueous solution was adopted as the optimal eluting solution because the recoveries were the highest (about $90 \%$ ) at that concentration. However, the elution by $1 \mathrm{~mL}$ of a $60 \%$ methanol aqueous solution was insufficient for the analysis 


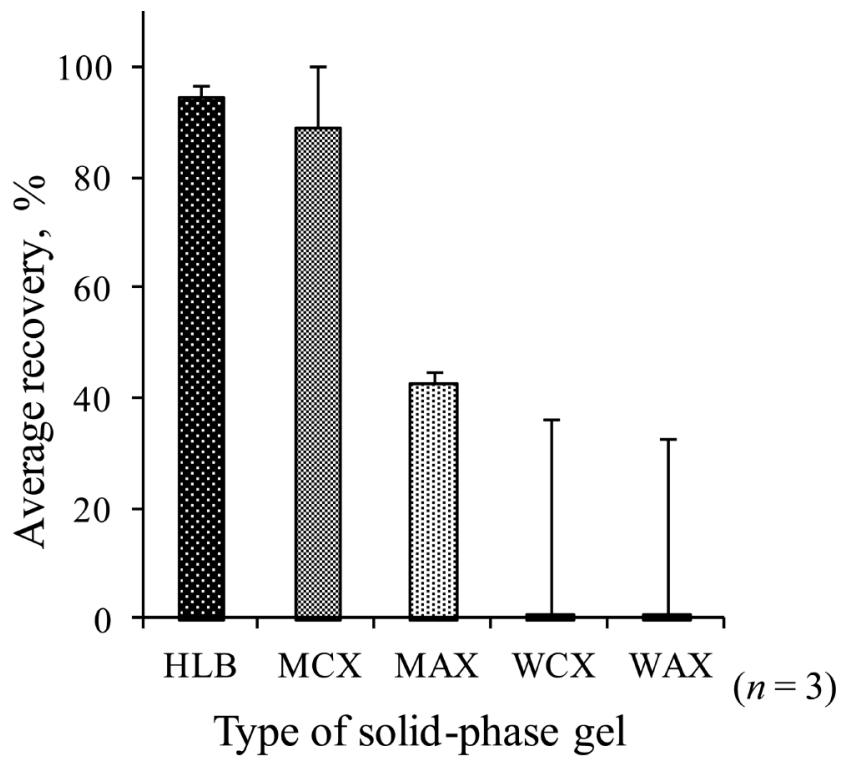

Fig. 4 Effect of the type of solid-phase gel used on the recovery of VCM after SPDE. Washing solution and eluting solution (MCX and WAX) consisting of $2 \%$ formic acid aqueous solution and $5 \%$ ammonia methanol solution, respectively. Washing solution and eluting solution (MAX and WCX) consisting of 5\% ammonia aqueous solution and 2\% formic acid methanol solution, respectively. Other operations were performed according to the method described in the text above.

of a real sample. Two milliliters of a $60 \%$ methanol aqueous solution was found to be sufficient for the elution of VCM from a real sample.

Next, the pooled serum was diluted with water and purified by SPDE by using the optimized experimental conditions for the validation of operability in serum analysis. Then, fine bubbles were generated inside the Captube ${ }^{\mathrm{TM}}$ when performing the first centrifugation of the sample. The surface action effect of the proteins contained in the pooled serum was considered to be the cause. When the solvent necessary for the next operation was added to the Captube ${ }^{\mathrm{TM}}$ in the presence of the bubbles, the bubbles overflowed, resulting in lower recovery. An extended period of time $(2-3 \mathrm{~h})$ was required for the bubbles to vanish. Therefore, the same operation was performed after the addition of a silicon antifoaming agent. ${ }^{24}$ Under these conditions, the bubbles in the Captube ${ }^{\mathrm{TM}}$ vanished. Importantly, the addition of the silicon antifoaming agent did not negatively affect the LC/MS measurements. The SPDE operability was increased by the addition of the silicon antifoaming agent.

\section{Recovery analysis by using serum samples}

The recovery analysis by using pooled serum samples was performed three times a day for five days. VCM was added at a concentration of $2 \mathrm{ng} / \mathrm{mL}$, at a concentration of $10 \mathrm{ng} / \mathrm{mL}$, and at a concentration of $100 \mathrm{ng} / \mathrm{mL}$ to the pooled serum. The sample was purified by SPDE and analyzed by LC/MS. Statistical analyses were performed using one-way analysis of variance (Table 1). The average recoveries of VCM from the pooled serum were $90.0 \%$ (when spiked at $2 \mathrm{ng} / \mathrm{mL}$ ), $90.8 \%$ (when spiked at $10 \mathrm{ng} / \mathrm{mL}$ ), and $98.6 \%$ (when spiked at $100 \mathrm{ng} / \mathrm{mL}$ ). The repeatabilities were $7.5,6.8$, and $2.8 \%$, respectively, and the intermediate precision values were $8.5,6.8$, and $7.0 \%$, respectively. It was confirmed that the proposed method has sufficient sensitivity and precision for TDM in a clinical setting.
Table 1 Recoveries of VCM in the pooled serum purified using SPDE or SPE

\begin{tabular}{ccccc}
\hline $\begin{array}{c}\text { Cleanup } \\
\text { method }\end{array}$ & $\begin{array}{c}\text { Amount } \\
\text { spiked/ } \\
\text { ng mL }\end{array}$ & $\begin{array}{c}\text { Average } \\
\text { recovery, } \\
\%\end{array}$ & $\begin{array}{c}\text { Repeatability, } \\
\%\end{array}$ & $\begin{array}{c}\text { Intermediate } \\
\text { precision, } \\
\%\end{array}$ \\
\hline SPDE & 2.0 & 90.0 & 7.5 & 7.5 \\
& 10 & 90.8 & 6.8 & 6.8 \\
SPE & 100 & 98.6 & 2.8 & 7.0 \\
& 10 & 31.1 & 9.1 & 17.9 \\
& 100 & 20.5 & 28.1 & 36.3 \\
\hline
\end{tabular}

$n=3 \times 5$ test.

(a)

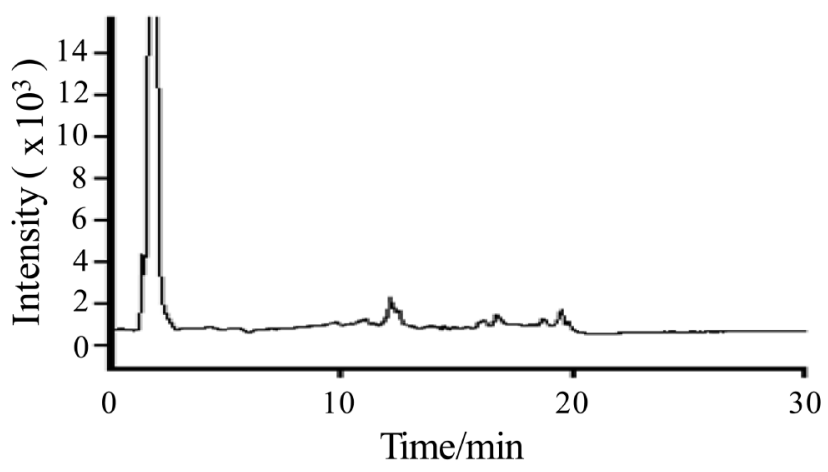

(b)

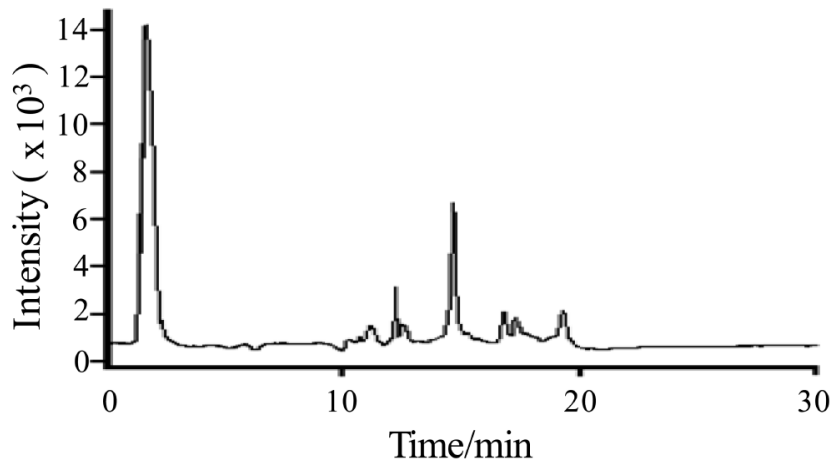

Fig. 5 Chromatograms of (a) a blank pooled serum and (b) a pooled serum spiked with $10 \mathrm{ng} / \mathrm{mL}$ VCM purified with SPDE.

Cleanup by SPDE yielded a good chromatogram that was little affected by impurities (Fig. 5). Subsequently, the influence of the matrix effect by SPDE was examined. Preliminarily, the blank eluate of pooled serum purified with SPDE was prepared. The blank eluate was measured by LC/MS after addition of VCM. As a result, matrix effects such as ion suppression and/or ion enhancement were seldom observed.

Comparison of operation time and recoveries between SPDE and cartridge-type SPE

The cleanup efficiencies of SPDE and cartridge-type SPE were compared. For cartridge-type SPE, a pooled serum sample was centrifuged for $10 \mathrm{~min}$ at $3000 \times g$ after deproteinization extraction by $10 \%$ TCA, and the supernatant was collected. This deproteinization procedure was repeated two times, and the two supernatants were combined. The supernatant was purified by cartridge-type SPE. The operation was performed by natural 
gravity filtration. If the flow rate was quick, the recovery decreased drastically. The operation time was about $2 \mathrm{~h}$ and the recovery was about $70 \%$. The low recovery of the SPE method compared with SPDE was attributed to the loss of VCM by coprecipitation during deproteinization. Subsequently, the pooled serum was purified by cartridge-type SPE after dilution with water, similar to the procedure proposed in this study. As a result, the recovery was extremely low (about 30\%) (Table 1), probably because the passage of the liquid through the cartridge was difficult because of the high viscosity of the extract.

On the other hand, the cleanup step using the SPDE method of this study was completed within about $15 \mathrm{~min}$, with a recovery of over $90 \%$. Therefore, quick cleanup was possible by SPDE, and the operation was not affected by the physical properties of the sample such as high viscosity compared with conventional cartridge-type SPE.

\section{Conclusions}

The recovery of VCM was improved to over $90 \%$, and the time to complete all the operations was shortened from $2 \mathrm{~h}$ to $15 \mathrm{~min}$ by using SPDE compared with cartridge-type SPE. In addition, SPDE could efficiently eliminate proteins without any other deproteinization procedure. The cleanup by SPDE yielded a chromatogram without any impurity. The average recoveries of VCM from the pooled serum were $90.0 \%$ (when spiked at $2 \mathrm{ng} / \mathrm{mL}$ ), $90.8 \%$ (when spiked at $10 \mathrm{ng} / \mathrm{mL}$ ), and $98.6 \%$ (when spiked at $100 \mathrm{ng} / \mathrm{mL}$ ). The repeatabilities were $7.5,6.8$, and $2.8 \%$, respectively, and the intermediate precision values were $8.5,6.8$, and $7.0 \%$, respectively. Using SPDE, matrix effects such as ion suppression and/or ion enhancement were seldom observed.

These results demonstrate that sample cleanup by SPDE is a useful method for the determination of VCM in serum, and therefore, its clinical application is expected.

\section{References}

1. N. Patel, M. P. Pai, K. A. Rodvold, B. Lomaestro, G. L. Drusano, and T. P. Lodise, Clin. Infect. Dis., 2011, 52, 969.

2. J. F. Mohr and B. E. Murray, Clin. Infect. Dis., 2007, 44, 1536.

3. R. Kullar, S. L. Davis, D. P. Levine, and M. J. Rybak, Clin. Infect. Dis., 2011, 52, 975.

4. M. N. Jeffres, W. Isakow, J. A. Doherty, S. T. Micek, and M. H. Kollef, Clin. Ther, 2007, 29, 1107.
5. R. L. Davis, A. L. Smith, and J. R. Koup, Ann. Intern. Med., 1986, 104, 285.

6. A female infant aged 1 month was exposed to ten times dose of VCM. Toe necrosis., YOMIURI ONLINE, 2013/8/22.

7. H. Kokubun, T. Kimura, and K. Yago, Yakugaku Zasshi, 2007, 127, 925.

8. C. Monchaud and P. Marquet, Clin. Pharmacokinet., 2009, $48,489$.

9. N. J. Saunders, S. V. Want, and D. J. Adams, J. Antimicrob. Chemother., 1995, 36, 411.

10. H. J. Peckman, R. E. Dupuis, W. T. Sawyer, K. L. R. Brouwer, and R. E. Cross, Ther. Drug Monit., 1996, 18, 647.

11. M. C. Verdier, O. Tribut, P. Tattevin, Y. L. Tulzo, C. Michelet, and D. Bentué-Ferrer, Antimicrob. Agents Chemother, 2011, 55, 4873.

12. M. Aravagiri, S. R. Marder, T. Van Putten, and K. K. Midha, J. Pharm. Sci., 1993, 82, 447.

13. P. J. Taylor, Ther. Drug Monit., 2004, 26, 215.

14. R. W. Sparidans, T. T. A. Ahmed, E. W. Muilwijk, M. E. B. Welzen, J. H. M. Schellens, and J. H. Beijnen, Life Sci., 2012, 905, 137.

15. D. F. Chollet, E. Castella, L. Goumaz, and G. Anderegg, $J$. Pharm. Biomed. Anal., 1999, 21, 641.

16. H. A. G. Niederländer, E. H. M. Koster, M. J. Hilhorst, H. J. Metting, M. Eilders, B. Ooms, and G. J. de Jong, J. Chromatogr. B, 2006, 834, 98.

17. M. M. Kushnir, J. Crossett, P. I. Brown, and F. M. Urry, J. Anal. Toxicol., 1999, 23, 1.

18. R. Saito, Y. Kikuchi, Y. Iwasaki, R. Ito, and K. Saito, in Proceedings of the 54th Symposium of the Pharmaceutical Society of Japan Kanto Branch, 2010, Tokyo, 107.

19. T. Zhang, D. G. Watson, C. Azike, J. N. A. Tettey, A. T. Stearns, A. R. Binning, and C. J. Payne, J. Chromatogr. B, 2007, 857, 352.

20. I. Baranowska, A. Wilczek, and J. Baranowski, Anal. Sci., 2010, 26, 755.

21. K. Inoue, Y. Mizuno, Y. Yoshimi, M. Nunome, T. Hino, K. Tsutsumiuchi, and H. Oka, J. Sep. Sci., 2008, 31, 3871.

22. P. K. Smith, R. I. Krohn, and G. T. Hermanson, Anal. Biochem., 1985, 150, 76.

23. R. E. Brown, K. L. Jarvis, and K. J. Hyland, Anal. Biochem., 1989, 180, 136.

24. M. Mita, "Food hygiene inspection guideline food additive analysis method in food 2003", 2003, Public Interest Incorporated Association Japan Food Hygiene Association, Japan, 295. 